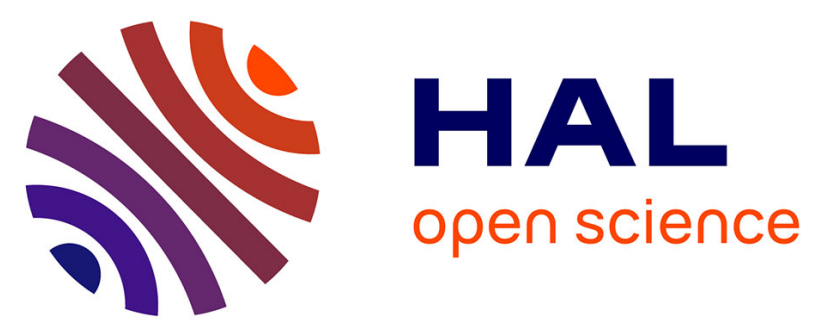

\title{
Chemistry deriving from OOQOOH radicals in alkane low-temperature oxidation: A first combined theoretical and electron-ion coincidence mass spectrometry study
} Frédérique Battin-Leclerc, Jérémy Bourgalais, Zied Gouid, Olivier Herbinet, Gustavo Garcia, Philippe Arnoux, Wang Zhandong, Luc-Sy Tran, Guillaume Vanhove, Laurent Nahon, et al.

\section{To cite this version:}

Frédérique Battin-Leclerc, Jérémy Bourgalais, Zied Gouid, Olivier Herbinet, Gustavo Garcia, et al.. Chemistry deriving from $\mathrm{OOQOOH}$ radicals in alkane low-temperature oxidation: A first combined theoretical and electron-ion coincidence mass spectrometry study. Proceedings of the Combustion Institute, 2021, 38 (1), pp.309-319. 10.1016/j.proci.2020.06.159 . insu-02918741

\section{HAL Id: insu-02918741 \\ https://hal-insu.archives-ouvertes.fr/insu-02918741}

Submitted on 14 Nov 2020

HAL is a multi-disciplinary open access archive for the deposit and dissemination of scientific research documents, whether they are published or not. The documents may come from teaching and research institutions in France or abroad, or from public or private research centers.
L'archive ouverte pluridisciplinaire HAL, est destinée au dépôt et à la diffusion de documents scientifiques de niveau recherche, publiés ou non, émanant des établissements d'enseignement et de recherche français ou étrangers, des laboratoires publics ou privés. 


\section{CHEMISTRY DERIVING FROM OOQOOH RADICALS IN ALKANE LOW-TEMPERATURE OXIDATION: A FIRST COMBINED THEORETICAL AND ELECTRON-ION COINCIDENCE MASS SPECTROMETRY STUDY}

Frédérique Battin-Leclerc ${ }^{1}$, Jérémy Bourgalais ${ }^{2}$, Zied Gouid ${ }^{3}$, Olivier Herbinet ${ }^{1}$, Gustavo Garcia ${ }^{4}$, Philippe Arnoux ${ }^{1}$, Zhandong Wang ${ }^{5}$, Luc-Sy Tran ${ }^{6}$, Guillaume Vanhove ${ }^{6}$, Laurent Nahon ${ }^{4}$, Majdi Hochlaf $^{3}$

${ }^{1}$ Laboratoire Réactions et Génie des Procédés, CNRS, Université de Lorraine, ENSIC, 1, rue Grandville, BP 20451, 54001 Nancy Cedex, France

${ }^{2}$ LATMOS/IPSL, UVSQ Université Paris-Saclay, Sorbonne Université, CNRS, Guyancourt, France

${ }^{3}$ Université Gustave Eiffel, COSYS/LISIS, 5 Bd Descartes 77454, Champs sur Marne, France ${ }^{4}$ Synchrotron SOLEIL, L'Orme des Merisiers, Saint-Aubin-BP 48, 91192 GIF-sur-Yvette Cedex, France

${ }^{5}$ National Synchrotron Radiation Laboratory, University of Science and Technology of China, Hefei, Anhui 230029, P. R. China

${ }^{6}$ Univ. Lille, CNRS, UMR 8522 - PC2A - Physicochimie des Processus de Combustion et de l’Atmosphère, F-59000 Lille, France

While there is consensus on the fact that OOQOOH radicals, produced by two oxygen additions from alkyl radicals, are the heart of the low-temperature oxidation of alkanes, the determination of the isomeric distribution and the quantification of their derived products (ketohydroperoxides and diones) are still a challenge. For the first time, heavy oxygenated products produced during alkane oxidation have been investigated using electron/ion coincidence mass spectrometry. The investigated prototype reaction is $n$-pentane oxidation carried out in a jet-stirred reactor (temperatures from 585 to $665 \mathrm{~K}$, pressure of 1.1 bar, lean mixture). Identification attempts were made for $m / z 100$ and 118 species using coincident mass-tagged Slow PhotoElectron Spectra obtained by electron-ion coincidence mass spectrometry combined with first principle computations, consisting in the determination of their adiabatic ionization energies and the Franck-Condon envelope of the photoionization spectra. 4hydroperoxypentan-2-one has been confirmed as the dominant obtained ketohydroperoxide, as predicted by up-to-date kinetic models. However, difficulties due to fragmentation has made 
impossible the identification of the ketohydroperoxides present in lower amounts. In parallel, $\mathrm{C}_{5} \mathrm{H}_{8} \mathrm{O}_{2}$ isomers were identified, showing the possible formation, in addition to diones, of species with a ketone and an enol function. In addition, we provide new information on the first steps of the fragmentation pathways of $\mathrm{C}_{5}$ ketohydroperoxides. From the shape of their corresponding peaks on mass spectra and the energy and temperature dependence of their signal, ions at $m / z 43,57$ and 85 have been identified as fragments from ketohydroperoxides. Taking into account these fragmentations lowers, by more than a factor of 10, the previously observed deviation between experiments and modeling for ketohydroperoxide mole fractions. The formation of the $\mathrm{C}_{1}-\mathrm{C}_{2}$ carboxylic acids, predicted from Korcek decomposition, was also observed, but with a favored formation of acetic acid versus formic acid that what was predicted for propane.

Keywords: Jet-stirred reactor; electron/ion coincidence mass spectrometry; $n$-pentane; ketohydroperoxides; photo-ionization fragmentation; Ab initio computations. 


\section{INTRODUCTION}

Hydroperoxides, especially ketohydroperoxides (KHPs), play a key role during the low-temperature $(500-800 \mathrm{~K})$ gas-phase oxidation of alkanes [1][2] (see a summary of the up-to-date chemistry understanding related to ketohydroperoxides in Fig. S1 in Supplementary Materials (SM)). The importance of ketohydroperoxides in triggering autoignition is due to the well-known branching reaction initiated by $\mathrm{O}-\mathrm{OH}$ bond breaking. However, competing non-branching reactions was recently discovered, such as the formation of carboxylic acids or of species including two ketone functions, called diones.

Photoionization mass spectrometry (PIMS) recently succeeded in detecting a wide range of alkyl, alkenyl, and ketohydroperoxides [2]. The first repeatable identification of ketohydroperoxide (KHP) was obtained using tunable synchrotron photoionization and molecular beam sampling [2]. However, the determination of the isomeric distribution of these elusive species has not been achieved yet. In addition, while the attempts of quantification of alkyl hydroperoxides lead to values consistent with modeling, astonishing deviations (e.g. above a factor 100 in [3]) are obtained for ketohydroperoxides, likely because the fragmentation of molecular ions is not taken into account [2]. In all these previous studies, diones were systematically found to be produced together with ketohydroperoxides.

Previous work in flames [4] and a Jet-Stirred Reactor (JSR) [5] evidenced an interesting potential of PIMS using the PhotoElectron PhotoIon COincidence (PEPICO) technique for identifying isomers through the energy analysis of the correlated photoelectron. Therefore, the first purpose of this paper is to analyze the coincident mass-tagged Threshold PhotoElectron Spectra (TPES [6][7]) of ketohydroperoxides and their related products measured during an investigation of $n$-pentane oxidation (lean mixtures with dilution in helium) in JSR. $n$-Pentane is a representative fuel of gasoline components, which was thoroughly studied recently through JSR experiments with gas chromatography [11] and PIMS [3] measurements leading to very satisfactory modeling [11].

While their formation pathway is still not fully understood, diones are currently considered as directly formed from KHP or as products of chemically activated Q $\mathrm{OOH}+\mathrm{O}_{2}$ reaction in parallel with the 
formation of KHP [2][8]. Recent JSR works investigating the oxidation of oxygenated molecules (dimethyl ether [8], tetrahydrofuran [10]) have documented the possibility of better quantifying KHPs based on their fragmentation. The second purpose of this paper is shedding light on the first steps of the unimolecular decomposition pathways undertaken by the $\mathrm{C}_{5} \mathrm{KHPs}$ derived from $n$-pentane. Finally, we show also how these $\mathrm{C}_{5}$ KHPs end up with the production of carboxylic acids such as acetic acid and formic acids.

\section{EXPERIMENTAL FACILITY}

The used set-up, based upon the undulator-based DESIRS VUV beamline [12] of synchrotron SOLEIL(see Fig. S2 (SM)), was described in [5]). The SAPHIRS end-station [13] is equipped with the double-imaging photoelectron/photoion (i²PEPICO) spectrometer DELICIOUS III [14]. The JSR mounted inside SAPHIRS is similar to that used in [11] (same geometry, heating devices and temperature control, and gas feeding). The reactor sphere includes a quartz nozzle with a 100- $\mu$ m hole in order to create the molecular beam and is connected to two concentric quartz tubes mounted into a flange carrying all the gas inlets and outlets, which is itself mounted on a 3-axes manipulator [5]. $n$-Pentane is provided by Sigma-Aldrich (purity $\geq 99 \%$ ) and the gases by Air Liquide.

After leaving the reactor, the molecular beam traverses two consecutive skimmers expanding further $\left(10^{-6}\right.$ mbar) towards the ionization chamber where it intersects the ionizing focused VUV beam of DESIRS [12]. The resulting electrons and cations are accelerated in opposite direction towards respectively a velocity map imaging and ion Time-Of-Flight (TOF) and imaging ion spectrometer [14]. The coincidence detection scheme enables mass tagging of the electron images, and the selection, via the correlated ion images, of the events corresponding to molecules having a net velocity along the molecular beam, discarding the residual background from the chamber. After Abel inversion of the electron images, one obtains threshold photoelectron spectra-by selecting photoelectrons with near zero kinetic energy while scanning the photon energy — on mass-tagged compounds originating from the reactor, with a typical mass resolution $\mathrm{m} / \Delta \mathrm{m}$ of the order of 1700 , and a typical electron energy resolution of about $20 \mathrm{meV}$. 


\section{CALCULATION METHODS}

The main aim of the present computations is to elucidate the structure of the molecular species formed in the first steps after oxidation of $n$-pentane and their subsequent VUV photoionization. Thus, we carried out geometry optimizations, followed by frequency computations on neutral and ionic species of $\mathrm{C}_{5}$ KHPs of $m / z=118$, the major $\mathrm{C}_{5} \mathrm{H}_{8} \mathrm{O}_{2}$ species at $\mathrm{m} / \mathrm{z}=100$ and formic acid. These geometry optimizations were done at the PBE0/aug-cc-pVDZ level as implemented in GAUSSIAN 09 [16]. Subsequently, we performed single point (SP) computations using the costly (R)CCSD(T)-F12/augcc-pVTZ method as implemented in MOLPRO 2015[17]. While PBE0/aug-cc-pVDZ(opt) level leads to AIEs off by more than $0.2 \mathrm{eV}$ for medium-sized molecules, the composite scheme PBE0/aug-cc$\mathrm{pVDZ}(\mathrm{opt}) / /(\mathrm{R}) \mathrm{CCSD}(\mathrm{T})-\mathrm{F} 12 / \mathrm{aug}-\mathrm{cc}-\mathrm{pVTZ}(\mathrm{SP})$ allows accurate derivation of these AIEs (to within a maximum of $\pm 0.02 \mathrm{eV}$ ) as discussed previously [18] (see a comparison of the values obtained with both methods in Table S1 (SM)). Afterwards, we simulated the vibrationally-resolved Franck-Condon photoelectron spectra (FCPS), for the ion ground state of the species listed in Table 1, as well as some of their conformers that most closely mirror their corresponding neutral (see conformers list and all calculated FC PESs (SM)) using the Time-Independent Adiabatic Hessian Franck-Condon (TI$\mathrm{AH} \mid \mathrm{FC})$ model as implemented in GAUSSIAN, as described in more details in [5].

Anharmonicity was not considered. Indeed, its effect on the simulated spectra would not be appreciable due to the limited experimental resolution $(20 \mathrm{meV})$ and the congestion of these spectra. Note that the simulated spectra treat only direct photoionization. While the contribution of autoionizing resonances, especially close to the AIEs, cannot be fully ignored, Cooper minima and shape resonances effects can be safely ruled out since they are associated with the ejection of fast electrons discriminated by the threshold techniques employed in this work. Nevertheless, it is commonly admitted that autoionization equally affects the TPES bands for medium-sized molecules (as those treated here) allowing hence direct comparison of the simulated and measured spectra.

In a second step, the origin bands were shifted in energy to coincide with the respective PBE0/aug-ccpVDZ(opt)//(R)CCSD(T)-F12/aug-cc-pVTZ(SP) AIEs. Finally, the Franck-Condon factors are 
convoluted with a $20 \mathrm{meV}$ bandwidth Gaussian profile in order to facilitate the comparison between the computed envelopes (PhotoElectron Spectra (PES)) and the measured TPES based on the experimental resolution. This approach already gave good results for understanding the structure of biological molecules up to $\mathrm{m} / \mathrm{z} 136$ [19]. In [5], the validity of this approach not considering anharmonic calculations was demonstrated for organic species up to $m / z$ 88. Concerning hydroperoxides, our calculations neglecting the torsional effect around the O-O bond lead to good agreement between experimental [5] and simulated PES as demonstrated by figure S8 (SM) for methyl hydroperoxide $(\mathrm{m} / \mathrm{z}, 48)$.

\section{RESULTS AND DISCUSSION}

The apparatus described above was used to study the oxidation of $n$-pentane between 585 to $665 \mathrm{~K}$, under quasi-atmospheric pressure, at a mean residence time of $3.00 \pm 0.02 \mathrm{~s}$, for a fuel initial mole fraction of 0.01 and equivalence ratios $(\phi)$ of $1 / 3$ and 0.5 .

\section{Mass spectrum}

Typical TOF Mass Spectra (TOF-MS) at fixed photon energies of 10.0 and $11.5 \mathrm{eV}$ obtained for the oxidation of $n$-pentane at $606 \mathrm{~K}$ are shown in Fig. 1.

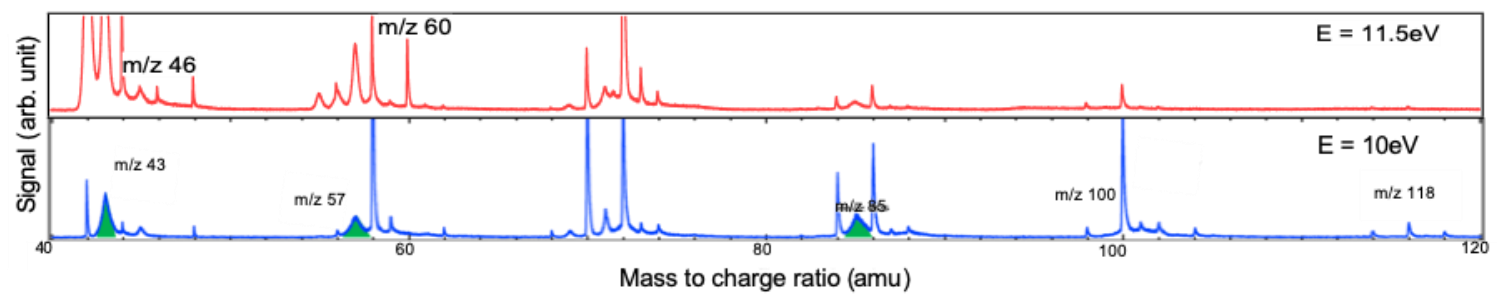

Figure 1: Mass spectra obtained during n-pentane oxidation at $606 \mathrm{~K}$ and $\phi=1 / 3$ for photon energies of 10.0 (green filled peaks correspond to fragments as discussed in the text) and $11.5 \mathrm{eV}$. The corresponding two-dimensional TOF-MS plotted as a function of $m / z$ and photon energy is displayed in Fig. S3 (SM). When compared with the TOF-MS recorded in [3] for a similar temperature (600 K) at $\phi=1$, all the $\mathrm{m} / z$ peaks between 50 and 90 were present in both spectra. In addition, the TOF-MS of Fig. 1 contains peaks at $m / z 100$ and 118 as that of [3]. The main difference between both TOF-MSs is that in the present TOF-MS, the fragments exhibit broader peak shapes while the parent 
ions appear as narrow peaks. This is due to the lower extraction field used in the present work which renders the experiment more sensitive to the Doppler broadening caused by the kinetic energy release in dissociative ionization. From the TOF spectrum depicted in Fig. 1, clearly $m / z, 43,57$ and 85 can be identified as daughter species and as such will be discussed further in the text.

\section{Attempts of determination of the isomeric distribution of ketohydroperoxides and diones}

As is shown in [5], the species indicated in the mass spectrum of Fig. 1 at $m / z<100$ can easily be identified according to their recorded PES (examples for acetaldehyde and acetic acid are given in Fig. S4 (SM). As already demonstrated [3] by comparison between ionization energies measured by PIMS at the Hefei synchrotron and theoretical calculations, $\mathrm{m} / \mathrm{z}, 100$ and 118 correspond to $\mathrm{C}_{5}$ diones and $\mathrm{C}_{5}$ ketohydroperoxides, respectively. In the following, an isomer identification attempt is done for $\mathrm{m} / \mathrm{z}$ 118 corresponding to KHPs and 100 corresponding to diones. The calculated theoretical spectra of Figure 2 were obtained from spectra derived from the Franck-Condon calculations given in SM, which were summed and weighted with the appropriate factors to adjust the experimental spectrum in the relevant photon energy range.

\section{2.a/ $\mathrm{m} / z 118$}

Table 1 presents the 10 possible $\mathrm{C}_{5} \mathrm{KHP}$ isomers corresponding to $\mathrm{m} / \mathrm{z} .118$, as well as the mole fractions calculated at $600 \mathrm{~K}$ using the detailed kinetic model of [11]. A cyclic ether hydroperoxide has also be considered (1-methyl,2-hydroperoxyfurane). Table 1 also gives the optimized structures of the $10 \mathrm{KHP}$ isomers and their AIEs as computed at the composite scheme PBE0(opt)/aug-ccpVDZ//(R)CCSD(T)-F12/aug-cc-pVTZ(SP) level. As established [18], the composite scheme is more reliable and we use its results for the assignment of the experimental spectra. Table 1 shows that all 10 KHPs have AIEs in the range $9.2-9.7 \mathrm{eV}$ - the range of the bands observed in the TPES spectrum associated with $m / z$ 118. Franck-Condon simulations from the optimized geometries were performed for these 10 KHPs and their previously mentioned conformers (see SM) to reproduce their PES.

The Fig. 2a. displays the experimental TPES measured for $m / z, 118$ and characterized by a slow rise after $8.8 \mathrm{eV}$, which is due to some geometry changes between the neutral and the cation leading to 
unfavorable FC factors. A significant signal drop after $9.4 \mathrm{eV}$ is also observed, indicating that fragmentation starts to occur from this photon energy in agreement with the energy dependence of the signal at $m / z 118$ displayed in Fig. 3a. Therefore, the analysis of the TPES may not be possible from a certain photon energy and only the signal below $9.8 \mathrm{eV}$ is considered in this work. Additionally, the simulated PES of 2,4KHP (4-hydroperoxypentan-2-one) displays a second intense peak between 9.8 and $10 \mathrm{eV}$ that is not present in the experimental PES (cf. Fig. 2a). Unfortunately, most of the KHPs present simulated PES above $9.8 \mathrm{eV}$ (see figures in SM), which would mean that the corresponding cation would be unstable. Thus, their formation could not be verified in this work.

5 KHPs (1,2KHP, 2,1KHP, 2,3KHP, 2,4KHP and 3,2KHP) and 1-methyl,2-hydroperoxyfurane have their PES below $9.8 \mathrm{eV}$ (see SM) and have been considered in the analysis of the measured $\mathrm{m} / \mathrm{z} 118$ TPES. A signal convolution, made between 9.4 and $9.8 \mathrm{eV}$ and presented in Fig. 2a, indicates that at least three hydroperoxides could be present, 1-methyl,2-hydroperoxyfurane, 1,2KHP and 2,4KHP, with a weight of $0.3,0.1$ and 1 respectively. Due to a somewhat low signal to noise ratio and an overlap between the simulated PES, the contribution of the three other KHPs could not be ruled out. However, the convoluted spectrum agrees well with the experimental TPES and 2,4KHP is the KHP formed in largest amounts amongst the 10 isomers, as predicted (see Table 1). In addition, this work suggests a more notable presence of 1,2KHP and 1-methyl,2-hydroperoxyfurane than predicted.

Note that the signal below $9.3 \mathrm{eV}$ could not be attributed to any calculated Frank-Condon PES, as shown in Fig. S9 (SM). This could be due to the excitation of more internal modes when reaching relatively high masses, as well as to the existence of conformers leading to spectroscopic congestion, which results in a lack of structure explaining the large difference between the observed TPES and simulations. However, the conformers have too high AIEs to support this assumption. Thus, the lower part of the measured TPES is probably due to the increasing internal modes at this mass especially low frequencies that may be populated leading to hot bands which are not considered in the simulated PES since they are calculated at $0 \mathrm{~K}$. Indeed, such a situation with an initial slow rise for the signal in the 
lower part of the spectrum, due to some geometry variation between the neutral and the cation, makes the PES more sensitive to hot band contributions.

Table 1: Adiabatic ionization energies (AIE ((R)CCSD(T)-F12/aug-cc-pVTZ method), eV) including PBE0/aug-cc-pVDZ zero point vibrational energy corrections, and simulated mole fractions (MF at $600 \mathrm{~K}$ using the model of [11] of the 10 possible $C_{5}$ molecular structures with a hydroperoxide and a ketone function ((KHP) i.e. $\mathrm{m} / \mathrm{z}=118$, KHPs in bold are those with the highest MF) obtained during n-pentane oxidation. We also give the AIE of the most probable $\mathrm{C}_{5} \mathrm{H}_{8} \mathrm{O}_{2}$ species and formic acid (see text).

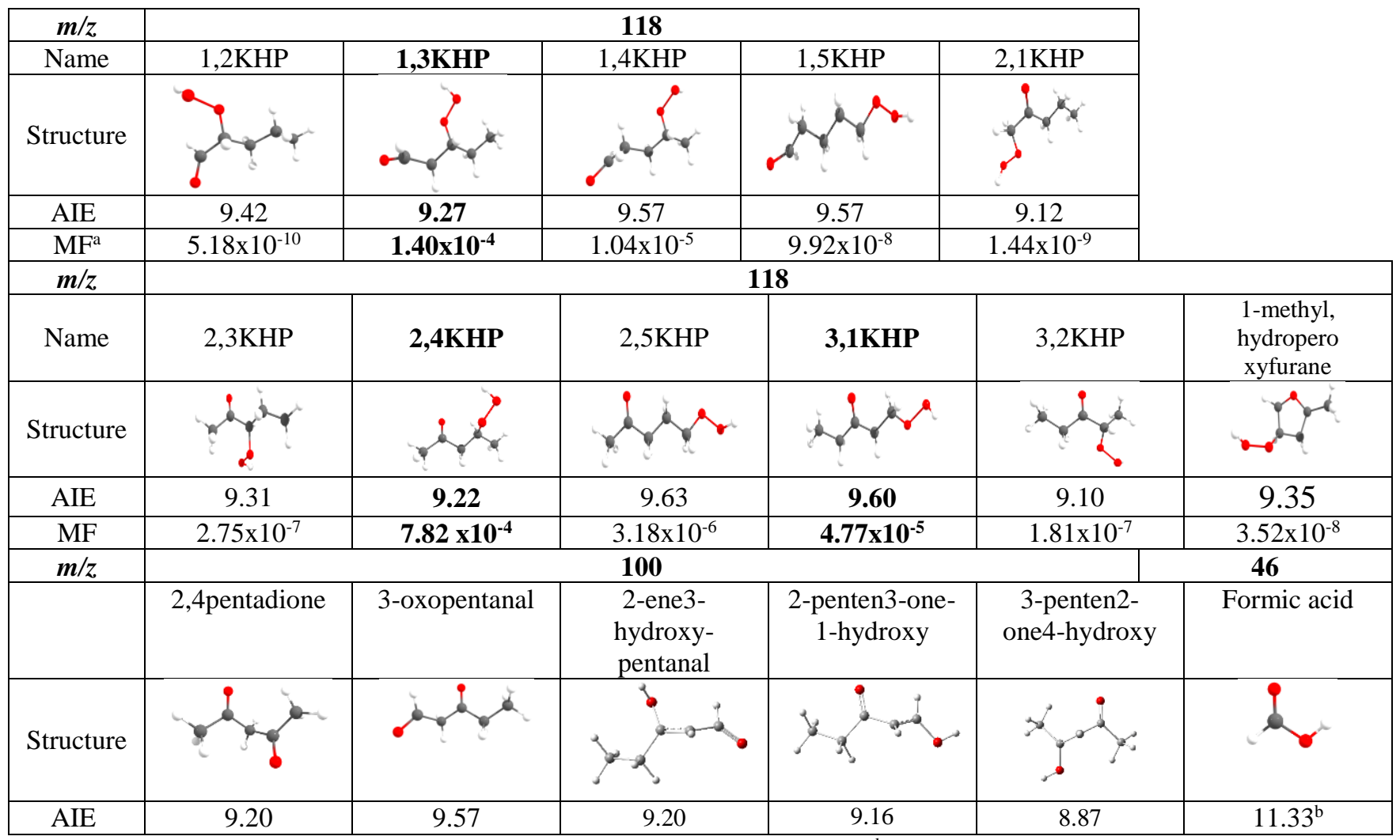

${ }^{\mathrm{a}}$ Mole fractions have been obtained from detailed kinetic model simulations, ${ }^{\mathrm{b}}$ To be compared to the value from the present experimental work as deduced from the PEPICO spectrum $(\mathrm{AIE}=11.29 \mathrm{eV})$ or that of [20] $(\mathrm{AEI}=11.31 \mathrm{eV})$. 

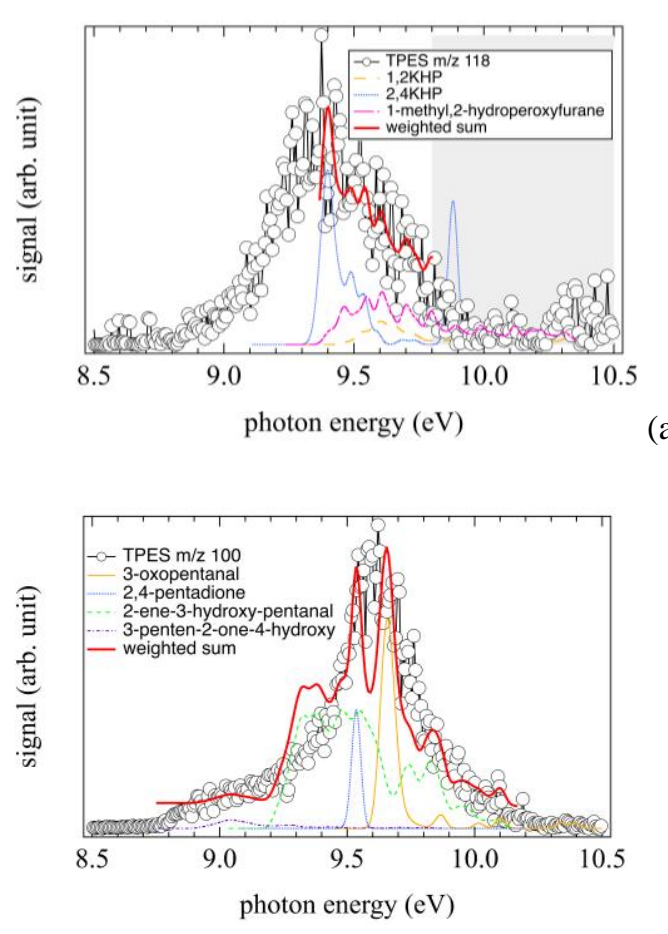

(b)

Figure 2: Comparison between the measured (under the conditions of Fig. 1) TPES spectra for m/z 118 (a) and 100 (b), and theoretically calculated PES based on the compounds listed in Table 1.
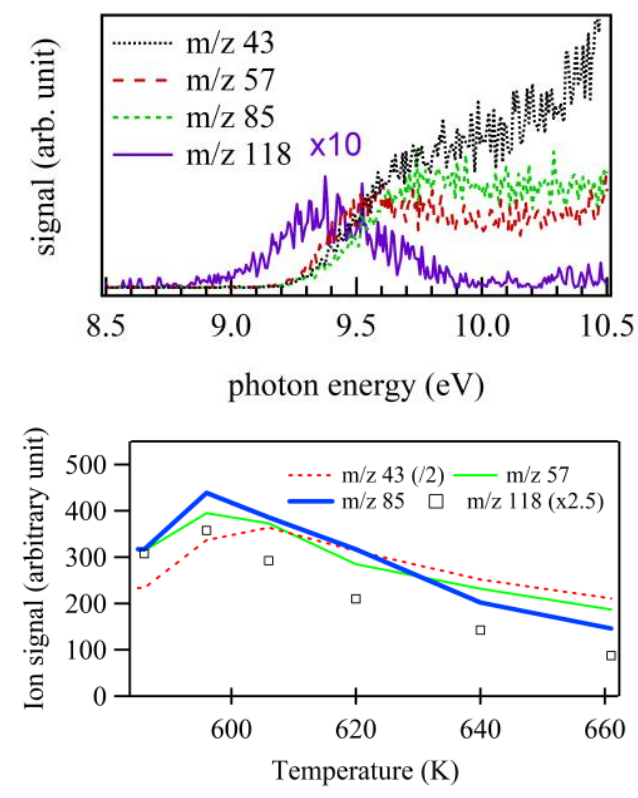

(a)

(b)

Figure 3: M/z 118, 85, 57 and 43 (under the conditions of Fig. 1): (a) TPES and (b) ion signal at $10.5 \mathrm{eV}$ as a function on temperature (numbers in the graphs indicate factors by which the signal are multiplied/divided).

KHP identification may also be attempted according to the main detected oxygenated products. The main expected low-temperature reaction pathways for the decomposition via $\mathrm{O}-\mathrm{OH}$ bond breaking of the three ketohydroperoxides of Table 1 predicted in highest amounts are displayed in Fig. 4. 


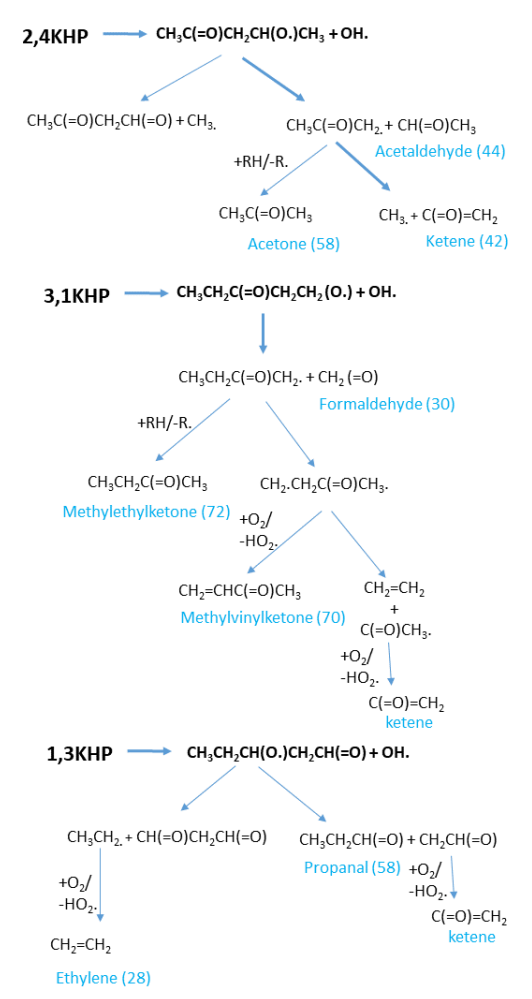

Figure 4: Main expected low-temperature reaction pathways from the major predicted KHPs deriving from n-pentane (Table 1); possible minor pathways towards acids or diones are not presented. Products in blue (with their $\mathrm{m} / \mathrm{z}$ ) were identified in [3] or [5].

All the oxygenated products, for which a formation channel is indicated in Fig. 4, have been quantified [3] or identified (formaldehyde, acetaldehyde, acetone, ketene, methylethylketone, methylvynilketone) [5], except $\mathrm{C}_{3}-\mathrm{C}_{4}$ diones. Formaldehyde was not discussed in [5], but an intense signal can be seen in Fig. S3 (SM) at $m / z, 30$ from an energy of $10.88 \mathrm{eV}$, the ionization energy of this $\mathrm{C}_{1}$ aldehyde [21].

$2 . b / \mathrm{m} / \mathrm{z} 100$

Table 1 presents the structure of the five most likely isomers at $m / z 100\left(\mathrm{C}_{5} \mathrm{H}_{8} \mathrm{O}_{2}\right.$ species, previously identified as being diones) arising from the same OOQOOH radical as 2,4KHP, the main identified ketohydroperoxides. We only considered the $\mathrm{C}_{5}$ species, in which the two oxygenated functions are separated by a carbon atom. For $m / z 100$ TPES spectrum, Fig. $2 \mathrm{~b}$ shows that it is mainly composed by bands that can be assigned to the contribution of 2,4-pentadione:3-oxopentanal:2-ene-3-hydroxypentanal:3-penten-2-one-4-hydroxy, since both the AIEs and the theoretical simulated spectra confirm 
this attribution, with the best fit obtained for a signal branching ratio of 0.6:1:0.6:0.04 with a reasonable uncertainty (uncertainty below $30 \%$ for the three dominant species). While it was not considered when starting this work since their formation has never been reported, the contribution of enols had to be taken into account to reproduce the spectrum shape below $9.5 \mathrm{eV}$. Their contribution in the signal indicates a non-negligible formation. Considering the possible formation of $\mathrm{C}_{5} \mathrm{H}_{8} \mathrm{O}_{2}$ products from the decomposition of the $\mathrm{C}_{5}-\mathrm{OOQOOH}$ radicals, the formation of enols is favored by their further stabilization in respect to their corresponding ketones because of the mesomeric effects. Despite the satisfactory overall agreement between experimental and simulated PES in fig. $2 \mathrm{~b}$, the deviation between $~ 9.2-9.4 \mathrm{eV}$ could indicate a missing identified species such as those containing a cyclic ether, which were not considered in the present calculations.

\section{Attempts of quantification of ketohydroperoxides}

As discussed by [2] and confirmed in the present work, an important fragmentation contribution needs to be considered when quantifying KHPs by PIMS. In light of the mass spectrum of Fig. 1 and of the identification of the major KHP, fragmentation patterns can be derived and used to refine KHP quantification.

\section{3.a/ Identification of KHP fragmentation patterns}

The mass spectrum of Fig. 1 clearly shows the presence of three fragments that could be attributed to the fragmentation of $\mathrm{C}_{5} \mathrm{KHP}: \mathrm{m} / z$ 43, 57 and 85. Fig. 3 displays the energy and temperature dependence of the signals at $m / z 118,85,57$ and 43 .

Fig 3(a) shows the TPES of the possible fragments and that of the $m / z, 118$ parent. The fragment curves share the same energy, around $9.2 \mathrm{eV}$, and their signal increases when that at $\mathrm{m} / \mathrm{z} 118$ decreases displaying similar curves. This supports our hypothesis where the KHPs at $m / z 118$ would lead to fragments at $m / z, 43,57$ and 85 . Note that $\mathrm{m} / \mathrm{z}, 43$ shows a different behavior above $10.3 \mathrm{eV}$, which coincides with the fragmentation onset of acetone [22]. In addition, the temperature profiles shown in Fig. 3 b clearly show a similar temperature dependence of $m / z, 118,85$ and 57 , further supporting the assumption that $m / z 85$ and 57 are fragments from dissociative ionization of $\mathrm{m} / \mathrm{z} 118$. The fragment at 
$m / z 85$ may correspond to the loss of $\mathrm{HOO}$ from 2,4 $\mathrm{KHP}+, 1,3 \mathrm{KHP}+$ as evidenced in Refs. [8] and [10]. While Hansen et al. [10] only found this fragmentation, Moshammer et al. [8] also established the possibility of losing $\mathrm{CO}$ and an $\mathrm{H}$ atom. In the present study, the mass spectra, such as that of Fig. 1, do not present any peak at $\mathrm{m} / z 117$ (H-loss) and only a very tiny one at $\mathrm{m} / \mathrm{z}, 90$ (CO loss). Concerning the $m / z, 43$ signal, Fig. $4 \mathrm{~b}$ shows that the position of the maximum is shifted by $10 \mathrm{~K}$, but it should be considered that all the three isomers of KHP displayed in Table 1 could fragment to contribute to the signal observed at $\mathrm{m} / \mathrm{z}$ 43. In addition, it can be attributed to the contribution of acetone to this channel, since the signals of Fig. $3 \mathrm{~b}$ were recorded at $\mathrm{hv}=10.5 \mathrm{eV}$, above the fragmentation onset of acetone.

Assuming the production of fragments at $\mathrm{m} / \mathrm{z} 85,57$ and 43 , the $2,4 \mathrm{KHP}^{+}$fragmentation patterns displayed in Fig. 5 can occur via the formation of an unstable fragment at $m / z$ 100, the same $m / z$ as diones.

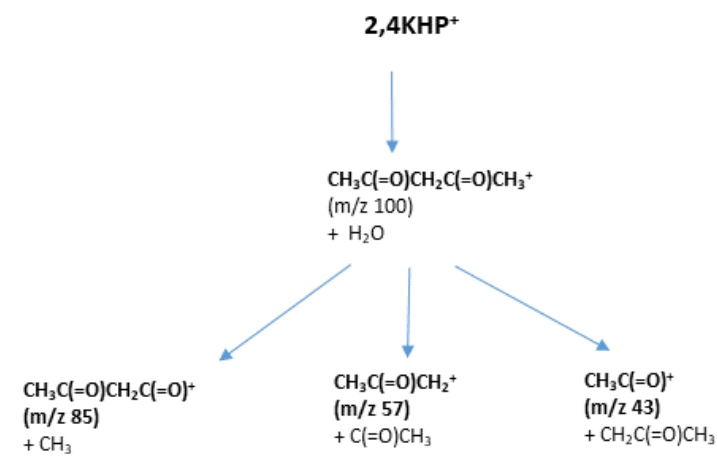

Figure 5: Fragmentation pathways proposed for 2,4-KHP ${ }^{+}$issued from the major KHP formed during n-pentane low-temperature oxidation.

These intermediates can be either the 2,4 pentanedione $^{+}$or the 3 -oxopentanal ${ }^{+}$, both species being identified in the present experimental study. Their formation from the corresponding KHPs can occur via the breaking of the $\mathrm{O}-\mathrm{O}$ weak bond together with the ejection of a water molecule. The further evolution of the $\mathrm{m} / \mathrm{z}$ ions leads to cationic or neutral $\mathrm{CH}_{2} \mathrm{C}(=\mathrm{O}) \mathrm{CH}_{2} \mathrm{CH}(=\mathrm{O}), \mathrm{CH}_{3}, \mathrm{CH}_{3} \mathrm{CH}_{2} \mathrm{C}(=\mathrm{O})$, $\mathrm{CH}_{2} \mathrm{CH}(=\mathrm{O})$ or their isomeric forms, including enol ones.

3.b/ Refining KHP quantification based on their fragmentation 
For quantifying KHPs from their PIMS experiments, Rodriguez et al. [3] used the formula $\left(\mathrm{S}_{\mathrm{i}}(\mathrm{T}, \mathrm{E}) / \mathrm{S}_{\mathrm{ref}}(\mathrm{T}, \mathrm{E})\right)=\left(\mathrm{X}_{\mathrm{i}}(\mathrm{T}) / \mathrm{X}_{\mathrm{ref}}(\mathrm{T})\right) \times\left(\sigma_{\mathrm{i}}(\mathrm{E}) / \sigma_{\text {ref }}(\mathrm{E})\right)$ taking as reference species (ref) propene quantified by gas chromatography, with $S_{i}(T, E)$ and $S_{r e f}(T, E)$, the signals of the species $i$ and ref at the temperature $T$ and at the photon energy $E, X_{i}(T)$ and $X_{\text {ref }}(T)$, the mole fraction of the species $i$ and ref at the temperature $\mathrm{T}, \sigma_{\mathrm{i}}(\mathrm{E})$ and $\sigma_{\mathrm{ref}}(\mathrm{E})$, the photoionization cross-section of the species $\mathrm{i}$ and ref at the photon energy E. In [3], the species were sampled using a capillary line, not through a molecular sampling, and mass discrimination factors were found equal to 1 in the range of studied $\mathrm{m} / \mathrm{z}$.

In light of the proposed fragmentation patterns, different reasons can be suggested to explain the observed 125-fold factor between experimental [3] and simulated mole fractions using the model of [11], while good agreement was found for other species. The quantification of fragmenting species remains difficult and has not been often addressed in literature. However, following the assumption made by Moshammer et al. [9], which led to experimental KHP mole fraction in good agreement with modeling, we used the following approach: if fragmentation is considered, $\mathrm{S}_{\mathrm{i}}(\mathrm{T})$ should not anymore be the single signal at $m / z, 118$, but the sum of signals of the parent and all daughter ions.

Considering that $m / z 85,57$ and 43 are all the possible fragments of ketohydroperoxides and that all the dissociative ionizations have been taken into account, the results of [3] can be reevaluated. According to [3], the signal height at m/z 118 contributes to only $8 \%$ in the sum of the daughter and parent ion signal height, and that at $\mathrm{m} / \mathrm{z} 85, \mathrm{~m} / \mathrm{z} 57$, and $\mathrm{m} / \mathrm{z} 43$ to $44 \%, 16 \%$, and $32 \%$, respectively. If the above-described fragmentation is considered, the calculated ketohydroperoxides mole fraction is 12 times higher than without considering the fragmentation. Note that this is a maximum value, since the fragment at $\mathrm{m} / \mathrm{z}, 43$ may possess several possible parent ions. While fragmentation has a very significant effect, it does not fully explain alone the observed deviation (a factor of 125).

Another experimental contribution to the error budget should also be taken into account: an error in the KHP photoionization total cross-section $\sigma_{\mathrm{i}}(\mathrm{E})$. This cross-section was estimated by [3] using group additivity method as being equal to $14.59 \mathrm{Mb}$ at $10.6 \mathrm{eV}$ (i.e. an energy between 1 and $1.3 \mathrm{eV}$ above 
the KHP ionization energies). This value is certainly too high when compared to values recently published for similar KHPs:

- $\quad 6.56 \mathrm{Mb}$ for hydroperoxymethyl formate at $11.5 \mathrm{eV}$, an energy $1.5 \mathrm{eV}$ above its ionization energy [8],

- between 3.3 and $8.3 \mathrm{Mb}$ for the different KHP isomers issued from tetrahydrofuran at an energy $1.5 \mathrm{eV}$ above their ionization energies [10].

Note that in the present case at least three isomers with potentially different cross-sections have to be taken into account. This means that as much as an error of a factor of three could be considered in the cross-section estimation. Considering both the error due to fragmentation and that to cross-section estimation, the remaining discrepancy between experiments and kinetic modeling could be around only a factor of three. Even if the kinetic pathways leading to ketohydroperoxides are quite trustable, the kinetics involved in the decomposition of these elusive intermediate is not so well determined [2]. Especially the rate constants for their major decomposition involving the $\mathrm{O}-\mathrm{OH}$ bond breaking rely on a single 1982 experimental measurement reviewed as shown by [2].

\section{Identification of carboxylic acid}

A last information about the chemistry of $\mathrm{C}_{5} \mathrm{KHP}$ is given by the mass spectrum obtained at an energy of $11.5 \mathrm{eV}$ (Fig. 1), where notable peaks at $\mathrm{m} / \mathrm{z} 46$ and 60 can be seen. As it was already shown (see fig. S3 (SM)), m/z 60 corresponds to acetic acid and was previously detected by gas chromatography [11] with a selectivity above 5\% in fuel-lean mixtures under the same temperature conditions as in Fig. 1. M/z 46 can be attributed to formic acid and was already observed during the oxidation of oxygenated fuel (e.g. dimethyl ether) and neo-pentane, but not yet from linear alkanes [2], while its formation from KHP together with acetic acid can occur from the Korcek mechanism as proposed in [23]. Indeed, Table 1 shows that the presently measured and computed AIE for $m / z=46$ species are close to the established AIE for formic acid. In addition, the computed PES for $\mathrm{HCOOH}$ (See SM) is similar to the He I PES spectrum of [20]. 
In order to see if the kinetic data calculated for propane [26] can be used for acid prediction in the case of $n$-pentane, Fig. 6 presents the ratios between the measured mole fraction of formic and acetic acids in the present study compared to the ratios calculated using the kinetic data calculated by [26]. This figure shows that, when $n$-pentane is used as fuel, the formation of acetic acid compared that of formic acid is about five times higher than in what could be computed using the kinetic data related to propane. The structure of the fuel has a significant influence on the prediction of acid formation. Therefore, fuel specific kinetic data need to be calculated if an accurate modeling of acid formation is expected.

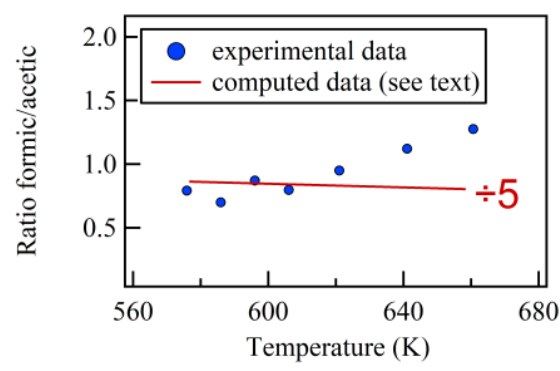

Figure 6: Ratio between the mole fraction of formic and acetic acids (under the conditions of Fig.

1): experimental (using cross sections from [24][25], respectively) and computed based on the reaction branching ratio of [26] (values divided by 5) values.

\section{CONCLUSIONS}

Electron-ion coincidence mass spectrometry has been used to revisit the JSR oxidation of $n$-pentane, confirming that $2,4 \mathrm{KHP}$ is the $\mathrm{C}_{5}$ ketohydroperoxide produced in highest amounts. An unexpected interesting results was the notable formation of $\mathrm{C}_{5}$ compounds with an enol and a ketone function together with that of diones. However, the important fragmentation (for the high binding energy part of the PES) has prevented more progresses in identifying ketohydroperoxides. In parallel, it was also demonstrated that considering their fragmentation may reconciliate model predictions and experimental measurements for these ketohydroperoxides. Although the assumptions involved prevent us from making a categorical statement on the precise mole fractions, we do demonstrate that the decrease of KHP concentration when compared to models, is compatible with the presence of dissociative ionization processes. Finally, the measurement of formic and acetic acids demonstrates the need to consider the fuel structure in attempts of predicting acid formation. 


\section{ACKNOWLEDGEMENT}

We are grateful to the whole SOLEIL staff for smoothly running the facility under project 20180021.

We warmly thank J.-F. Gil for his technical help around the SAPHIRS experiment. M.H. acknowledge the use of the computing center MésoLUM of the LUMAT research federation (FR LUMAT 2764).

J.B. thanks the ERC Starting Grant PRIMCHEM, grant agreement $\mathrm{n}^{\circ} 636829$ for financial support.

Z. Wang was supported by the National Natural Science Foundation of China (51976208).

\section{SUPPLEMENTARY MATERIAL:}

\section{The SM directory contains:}

\section{1 supplementary description file including:}

1/ Summary of the mechanism related to ketohydroperoxides (KHP) according to [2]

2/ Photograph and scheme of the experimental apparatus with obtained photoelectron and photoion images

3/ Typical Time-of-flight mass spectrum plotted as a function of $\mathrm{m} / \mathrm{z}$ and photon energy

4/ Example of lighter species identification according to [5]

5/ Comparison between AIEs calculated by at the PBE0/aug-cc-pVDZ level and with the (R)CCSD(T)-F12/aug-cc-pVTZ method

5/ Conformers considered for KHPs

6/ Calculated Franck-Condon photoelectron spectra

7/ Comparison between experimental [5] and newly simulated PES related to methylhydroperoxide $(\mathrm{m} / \mathrm{z}$ 48)

8) PES of the $10 \mathrm{C}_{5}$ KHPs

9/ TPES and PIE of formic carboxylic acid 


\section{REFERENCES}

[1] H. Curran, Proc. Developing detailed chemical kinetic mechanisms for fuel combustion, Proc. Combust. Inst. 37 (2019) 57-81.

[2] Z. Wang, O. Herbinet, N. Hansen, F. Battin-Leclerc, Exploring hydroperoxides in combustion: History, recent advances and perspectives, Prog. Energ. Combust. Sci. 73 (2019) 132-181.

[3] A. Rodriguez, O. Herbinet, Z. Wang, F. Qi, C. Fittschen, P.R. Westmoreland, F. Battin-Leclerc, Measuring hydroperoxide chain-branching agents during n-pentane low-temperature oxidation, Proc. Combust. Inst. 36 (2017) 333-342.

[4] D. Felsmann, K. Moshammer, J. Krüger, A. Lackner, A. Brockhinke, T. Kasper, T. Bierkandt, E. Akyildiz, N. Hansen, A. Lucassen, P. Oßwald, M. Köhler, G.A. Garcia, L. Nahon, P. Hemberger, A. Bodi, T. Gerber, K. Kohse-Höinghaus, Electron ionization, photoionization and photoelectron/photoion coincidence spectroscopy in mass-spectrometric investigations of a lowpressure ethylene/oxygen flame, Proc. Combust. Inst. 35 (2015) 779-786.

[5] J. Bourgalais, O. Herbinet, G. Garcia, G. Gouid, P. Arnoux, Z. Wang, L.S. Tran, G. Vanhove, M. Hochlaf, L. Nahon, F. Battin-Leclerc, Isomer-sensitive characterization of low temperature oxidation reaction products by coupling a jet-stirred reactor to an electron/ion coincidence spectrometer: case of $n$-pentane, Phys. Chem. Chem. Phys. 22 (2020) 1222-1241.

[6] J.C. Poully, J.P. Schermann, N. Nieuwjaer, F. Lecomte, G. Grégoire, C. Desfrançois, G.A. Garcia, L. Nahon, D. Nandi, L. Poisson, M. Hochlaf, Photoionization of 2-pyridone and 2hydroxypyridine, Phys. Chem. Chem. Phys. 12 (2010) 3566-3572.

[7] M. Briant, L. Poisson, M. Hochlaf, P. de Pujo, M.-A. Gaveau, B. Soep, Ar 2 Photoelectron Spectroscopy Mediated by Autoionizing States, Phys. Rev. Lett. 109 (2012) 193401.

[8] A.J. Eskola, I.O. Antonov, L. Sheps, J.D. Savee, D.L. Osborn, C.A. Taatjes, Time-resolved measurements of product formation in the low-temperature $(550-675 \mathrm{~K})$ oxidation of neopentane: a probe to investigate chain-branching mechanism, Physical Chemistry Chemical Physics. 19 (2017) 13731-13745. 
[9] K. Moshammer, A.W. Jasper, D.M. Popolan-Vaida, Z. Wang, V.S. Bhavani Shankar, L. Ruwe, C.A. Taatjes, P. Dagaut, N. Hansen, Quantification of the keto-hydroperoxide $\left(\mathrm{HOOCH}_{2} \mathrm{OCHO}\right)$ and other elusive intermediates during low-temperature oxidation of dimethyl ether, J. Phys. Chem. A. 120 (2016) 7890-7901.

[10] N. Hansen, K. Moshammer, A.W. Jasper, Isomer-Selective Detection of Keto-Hydroperoxides in the Low-Temperature Oxidation of Tetrahydrofuran, J. Phys. Chem. A. 123 (2019) 82748284.

[11] J. Bugler, A. Rodriguez, O. Herbinet, F. Battin-Leclerc, C. Togbé, G. Dayma, P. Dagaut, H. Curran, An experimental and modelling study of $n$-pentane oxidation in two jet-stirred reactors: The importance of pressure-dependent kinetics and new reaction pathways, Proc. Combust. Inst. (2017) 441-448.

[12] L. Nahon, N. de Oliveira, G.A. Garcia, J.-F. Gil, B. Pilette, O. Marcouillé, B. Lagarde, F. Polack, DESIRS: A state-of-the-art VUV beamline featuring high resolution and variable polarization for spectroscopy and dichroism at SOLEIL, J. Synchrotron Radiat. 19 (2012) 508-520.

[13] X. Tang, G.A. Garcia, J.-F. Gil, L. Nahon, Vacuum upgrade and enhanced performances of the double imaging electron/ion coincidence end-station at the vacuum ultraviolet beamline DESIRS, Rev. Sci. Instrum. 86 (2015) 123108.

[14] G.A. Garcia, B.K. Cunha de Miranda, M. Tia, S. Daly, L. Nahon, DELICIOUS III: A multipurpose double imaging particle coincidence spectrometer for gas phase vacuum ultraviolet photodynamics studies, Rev. Sci. Instrum. 84 (2013) 053112.

[15] J.-F. Gil, L. Nahon, Vacuum upgrade and enhanced performances of the double imaging electron/ion coincidence end-station at the vacuum ultraviolet beamline DESIRS, Rev. Sci. Instrum. 86 (2015) 123108.

[16] M. J. Frisch, G. W. Trucks, H. B. Schlegel, G. E. Scuseria, M. A. Robb and J. R. Cheeseman, Gaussian 09, Revision A02, Wallingford CT, 2016. 
[17] Q. Ma, H.-J. Werner, Explicitly correlated local coupled-cluster methods using pair natural orbitals. Wiley Interdis. Rev.: Comput. Mol. Sci. 8 (2018) e1371.

[18] M. Hochlaf, Advances in spectroscopy and dynamics of small and medium sized molecules and clusters, Phys. Chem. Chem. Phys. 19 (2017) 21236-21261.

[19] H.Y. Zhao, K.-C. Lau, G.A. Garcia, L. Nahon, S. Carniato, L. Poisson, M. Schwell, M.M. AlMogren, M. Hochlaf, Unveiling the complex vibronic structure of the canonical adenine cation, Phys. Chem. Chem. Phys. 20 (2018) 20756-20765.

[20] S. Leach, M. Schwell, D. Talbi, G. Berthier, K. Hottmann, H.W. Jochims, H. Baumgeartel, He I photoelectron spectroscopy of four isotopologues of formic acid: $\mathrm{HCOOH}, \mathrm{HCOOD}, \mathrm{DCOOH}$ and DCOOD. Chemical Physics 286 (2003) 15-43.

[21] NIST Chemistry Webbook; NIST Standard Reference Database 69; NIST, Gaithersburg, MD, 2005: http://webbook.nist.gov/chemistry.

[22] E.E. Rennie, A.-M. Boulanger, P.M. Mayer, D.M.P. Holland, D.A. Shaw, L. Cooper, L.G. Shpinkova, A Photoelectron and TPEPICO Investigation of the Acetone Radical Cation, J. Phys. Chem. A. 110 (2006) 8663-8675.

[23] A. Jalan, I.M. Alecu, R. Meana-Pañeda, J. Aguilera-Iparraguirre, K.R. Yang, S.S. Merchant, D.G. Truhlar, W.H. Green, New Pathways for Formation of Acids and Carbonyl Products in Low-Temperature Oxidation: The Korcek Decomposition of $\gamma$-Ketohydroperoxides, J. Am. Chem. Soc. 135 (2013) 11100-11114.

[24] S. Leach, M. Schwell, S. Un, H.W. Jochims, H. Baumgärtel, VUV absorption spectrum of acetic acid between 6 and $20 \mathrm{eV}$, Chemical physics, 321(1-2) (2006) 159-170.

[25] T.A. Cool, J. Wang, K. Nakajima, C.A. Taatjes, A. Mcllroy, Photoionization cross sections for reaction intermediates in hydrocarbon combustion, International Journal of Mass Spectrometry, 247(1-3) (2005) 18-27. 
[26] C.F. Goldsmith, M.P. Burke, Y. Georgievskii, S.J. Klippenstein, Effect of non-thermal product energy distributions on ketohydroperoxide decomposition kinetics, Proceedings of the Combustion Institute. 35 (2015) 283-290. 